\title{
LA CALIBRACIÓN INSTRUMENTAL DE REGISTROS CLIMÁTICOS DOCUMENTALES. APROXIMACIÓN METODOLÓGICA A RESOLUCIÓN ANUAL PARA EL CASO DE LA PRECIPITACIÓN EN BARCELONA (1521-1989)
}

Mariano Barriendos

Juan Carlos Peña

Javier Martín Vide

\section{RESUMEN}

La información proxy-data procedente de documentación manuscrita conservada en archivos históricos españoles es especialmente abundante y detallada en lo referente al régimen de precipitaciones. Diferentes métodos de tratamiento estadístico pueden permitir la disponibilidad de estos datos en análisis climáticos. Uno de ellos es la calibración de proxy-data con registros meteorológicos instrumentales de precipitación. Este artículo presenta un ensayo a resolución anual para el caso de Barcelona.

Palabras clave: calibración, climatología histórica, índices hídricos, proxy-data, regresión.

\section{ABSTRACT}

Proxy-data information obtained from manuscript documentation of Spanish historical archives is rich and detailed concerning especially rainfall regime. Different statistical treatment methodologies allow data availability for climatic analysis. One of them is calibration of proxy-data with instrumental meteorological records of rainfall. This paper presents a first proof for the case study of Barcelona in yearly resolution.

Key words: calibration, historical climatology, hydric indices, proxy-data, regression.

\section{Introducción}

La climatología histórica aún es una especialidad reciente en España. Las iniciativas puntuales y discontinuas no han fraguado una primera tesis doctoral hasta finales del siglo XX (BARRIENDOS, 1994). La obtención de informaciones climáticas cuantifica- 
bles pero con un nivel de detalle modesto hace necesaria la búsqueda de métodos de tratamiento de los datos que ofrezca mejoras en la interpretación de los resultados. El desarrollo de índices climáticos es una de las líneas de investigación que está actualmente vigente a diferentes escalas espacio-temporales de análisis (PFISTER, 1988; GLASER, 1991; FRENZEL, 1994).

Uno de los objetivos perseguidos y necesarios como etapa de la investigación es la calibración de las series procedentes de documentación histórica con series procedentes de registros meteorológicos instrumentales. Esta actividad tiene referentes en España dentro de la dendroclimatología (CREUS, FERNÁNDEZ CANCIO y MANRIQUE, 1996, 1997). Los ensayos metodológicos aquí presentados emplean series proxy-data a resolución anual para Catalunya entre los siglos XVI y XIX y una serie meteorológica instrumental de las más antiguas de Europa, con registros pluviométricos mensuales desde 1786.

\section{Datos utilizados}

\subsection{Información climática procedente de fuentes documentales (proxy-data)}

\subsubsection{Localización y características de las series proxy-data}

La aportación de series de datos climáticos a partir de fuentes documentales históricas en el presente trabajo procede de una investigación desarrollada en el período 1989-1994 con el fin de identificar y caracterizar la dinámica climática de Catalunya entre los siglos XV y XIX (BARRIENDOS, 1994).

Los puntos de observación fueron seleccionados en función de la calidad de conservación y contenidos de sus fondos documentales, fundamentalmente municipales y eclesiásticos. Por otra parte, se pretendió que las localidades objeto de estudio fueran representativas de los diferentes sectores geográficos que caracterizan el medio físico catalán: como series básicas se seleccionaron cuatro localidades costeras o cercanas al litoral equidistribuidas latitudinalmente (Girona, Barcelona, Tarragona y Tortosa), una localidad de montaña (Seu d'Urgell) y otras dos interiores (Vic y Cervera). Las series con información sobre inundaciones son más abundantes y permiten complementar las anteriores hasta llegar a una densidad de información aceptable (ver figura 1).

La recopilación de información se realizó casi en su totalidad en archivos históricos de ámbitos municipal y eclesiástico, donde se accedía a libros de actas o resoluciones de los órganos de gobierno de dichas entidades. La necesidad de establecer series continuas obligó a una consulta sistemática de series documentales, que suelen comprender varios centenares de volúmenes o legajos de documentos manuscritos.

En síntesis, la actividad recopiladora requirió el trabajo en 26 archivos, con la consulta de 112 series documentales y un número final de 2.130 volúmenes (ver detalle por poblaciones en tabla 1 ).

La objetivización y cuantificación de la información obtenida en fuentes documentales históricas se ha centrado básicamente en la elaboración de series de rogativas, con especial énfasis en las rogativas ocasionadas por la sequía, y los episodios de precipitaciones intensas que provocan inundaciones en cursos fluviales permanentes o intermitentes.

Las series que se han podido reconstruir (ver tablas 1 y 2) configuran una muestra representativa de los diferentes ámbitos geográficos catalanes, así como de los cursos fluviales de diferentes procedencias y regímenes que allí pueden encontrarse. Su cobertura temporal 


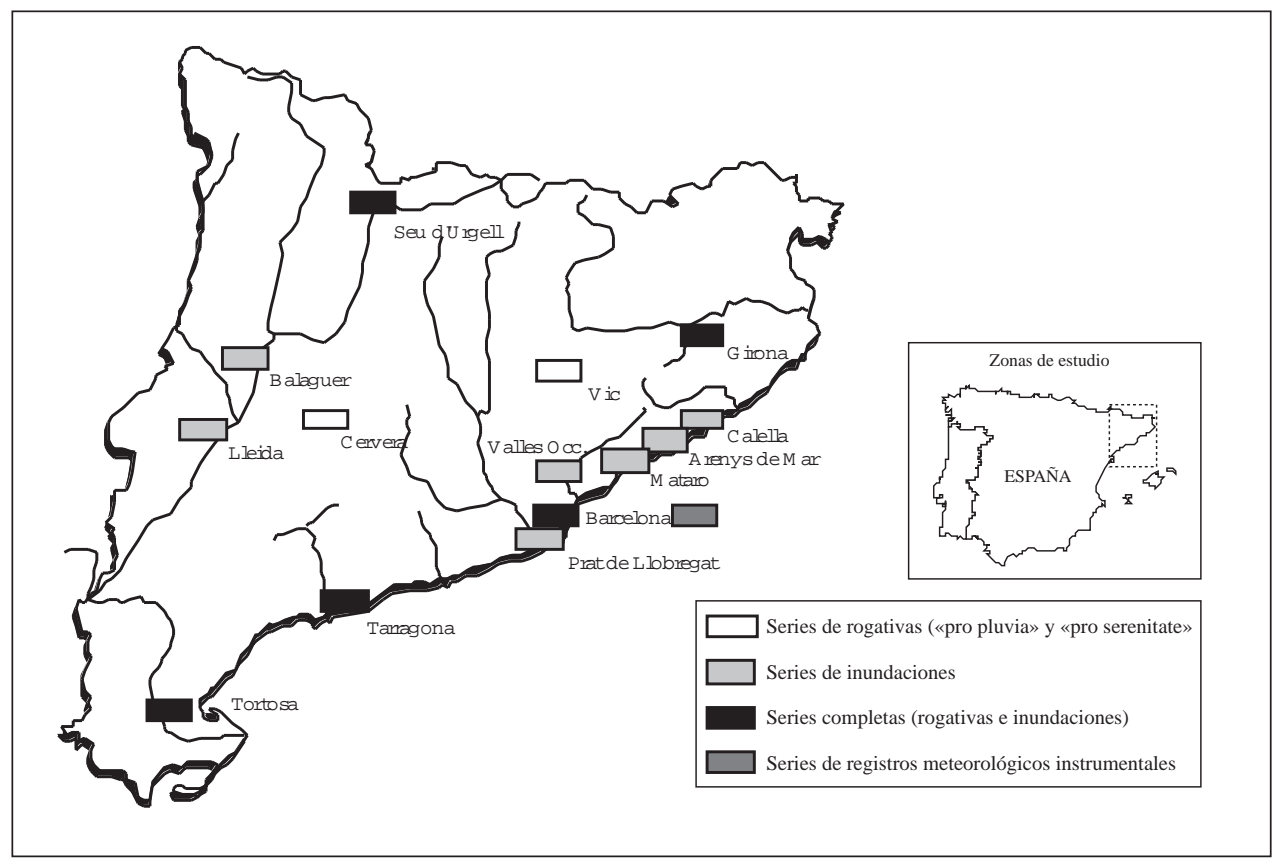

FIGURA 1. Localización geográfica de las series proxy-data empleadas en el estudio.

\begin{tabular}{|l|c|c|c|}
\hline Localidad & Archivos & Series documentales & Volúmenes manuscritos \\
\hline Girona & 4 & 12 & 428 \\
Barcelona & 7 & 45 & 354 \\
Reus/Tarragona & 4 & 10 & 410 \\
Tortosa & 2 & 8 & 354 \\
Seu d'Urgell & 1 & 2 & 48 \\
Vic & 2 & 6 & 93 \\
Cervera & 1 & 4 & 256 \\
Balaguer & 1 & 3 & 5 \\
Lleida & 1 & 4 & 4 \\
Calella & 1 & 7 & 39 \\
Arenys de Mar & 1 & 7 & 63 \\
Mataró & 1 & 4 & 76 \\
\hline \multicolumn{1}{|c|}{ Total } & 26 archivos & 112 series & 2130 volúmenes \\
\hline
\end{tabular}

TABLa 1. Fuentes documentales consultadas para la elaboración de los índices climáticos de Catalunya (Barriendos, 1994, actualizado). Las series de inundaciones del río Besòs y Llobregat proceden de fuentes bibliográficas (Sanz, 1983 y Codina, 1971, respectivamente) (ver bibliografía). 
también es considerable aunque en ningún caso se puede llegar a la Alta Edad Media con una disponibilidad óptima de datos.

Los criterios y métodos de trabajo aplicados han pretendido en todo momento la generación de series de datos que cumplieran unas características básicas mínimas. Respecto a las fuentes documentales que contenían la información útil, debían tener asegurada su continuidad temporal, homogeneidad y datación exacta de la misma (Le Roy Ladurie, 1967). También debía asegurarse la fiabilidad de los documentos consultados, incidiendo especialmente en la contemporaneidad de los hechos respecto al testimonio escrito que se ofrecía de los mismos (ALEXANDRE, 1987).

Respecto a las informaciones utilizadas, no se ha dispuesto de información climática directa. Se ha empleado información indirecta («proxy-data») tanto para los déficits como para los excesos hídricos.

\subsubsection{La información sobre déficits hídricos}

En el primer caso, se ha empleado como información de base los registros documentales generados por las instituciones municipales y eclesiásticas en el momento de convocar ceremonias religiosas de rogativas «pro pluvia».

La credibilidad de estas ceremonias como manifestación de una alteración o variación meteorológica está garantizada por la participación de diferentes instituciones (gremiales, municipales y eclesiásticas) que analizaban la situación y deliberaban antes de tomar cualquier decisión. La fiabilidad de los documentos está fuera de duda por tratarse de libros de actas municipales o capitulares, de cuya autenticidad daba fe un notario.

Las rogativas como información cuantificable presentan el factor positivo de su rigidez formal, al presentar de forma general, por lo menos en el ámbito de los reinos de la monarquía hispánica, cinco tipos muy diferenciados de funciones religiosas según la intensidad o duración del episodio de sequía que se estaba registrando (ver tabla 2).

\begin{tabular}{|c|l|l|l|}
\hline Nivel & Grado & Tipo de ceremonia & Ambito \\
\hline I & Leve & Oración al intercesor & Intra Ecclesiam \\
II & Medio & Exposición del intercesor & Intra Ecclesiam \\
III & Grave & Procesión con el intercesor & Extra Ecclesiam, Intra Civitatem \\
IV & Muy grave & Inmersión del intercesor & Extra Ecclesiam, Intra Civitatem \\
V & Crítico & Peregrinación al intercesor & Extra Ecclesiam, Extra Civitatem \\
\hline
\end{tabular}

TABLA 2. Niveles de sequía según el tipo de ceremonia de rogativas empleado (Martín Vide y Barriendos, 1995).

Las series de proxy-data con rogativas «pro pluvia» constituyen sin duda el conjunto de informaciones climáticas más densas y continuas de que se dispone en el patrimonio histórico documental español. Las primeras investigaciones realizadas apuntan la posibilidad de generar series de calidad óptima en aquellas poblaciones que dispusieran de forma continuada de un mínimo de estructura institucional. El problema planteado hasta el momento es la limitada longitud de las series, que apenas pueden alcanzar los últimos momentos de 
la Baja Edad Media (excepcionalmente la población de Igualada dispone de un sistema estructurado de rogativas «pro pluvia» desde 1404). Las cronologías establecidas en la presente investigación así lo manifiestan (ver tabla 3), iniciándose las series entre finales del siglo XV y principios del siglo XVI.

\begin{tabular}{|l|c|c|}
\hline Localidad & Período & Años \\
\hline Girona & $1438-1881$ & 444 \\
Barcelona & $1521-1896$ & 376 \\
Reus/Tarragona & $1493-1874$ & 382 \\
Tortosa & $1565-1858$ & 294 \\
Seu d'Urgell & $1539-1843$ & 305 \\
Vic & $1568-1906$ & 339 \\
Cervera & $1484-1850$ & 367 \\
\hline
\end{tabular}

TABla 3. Series de rogativas «pro pluvia»y «pro serenitate» utilizadas en el índice general para Catalunya.

\subsubsection{La información sobre excesos hídricos}

Los episodios de lluvias intensas o continuadas tienen un reflejo documental indirecto a través de la información contenida en los documentos municipales describiendo los daños o problemas ocasionados por las inundaciones en las poblaciones respectivas.

La documentación histórica no registra siempre el fenómeno meteorológico que ocasiona las inundaciones. Sin embargo, se describen con exactitud los daños ocasionados, tanto en bienes públicos como privados. Este hecho facilita el establecimiento de una sucinta clasificación de las inundaciones según su intensidad.

Las crecidas simples, sin llegar a producir desbordamiento, apenas generan información escrita, salvo cuando producen algún problema por su aparición rápida (como en el caso de los cursos intermitentes). Sin embargo, las inundaciones pueden definirse en dos categorías según si el desbordamiento ocasiona solo molestias en la vida cotidiana de la población o si produce daños graves y destrucciones. En el primer caso, se consideran inundaciones extraordinarias, y en el segundo catastróficas.

Los inmuebles e infraestructuras que pueden servir de indicador son diversas. Las que más frecuentemente sufren los efectos de las inundaciones son las relacionadas con la obtención de recursos hidráulicos y las infraestructuras en general: puentes, molinos, presas, murallas, acequias, caminos, pero también todo tipo de edificios, instalaciones agrícolas y cultivos.

Los registros de inundaciones presentan una cobertura temporal vinculada al desarrollo urbanístico de la localidad. Por este motivo, la cobertura temporal de las series de inundaciones es desigual y vinculada a cada caso específico (ver tabla 4). Por ejemplo, es evidente la gran cobertura en localidades importantes, como Barcelona, Girona o Tortosa, desde el siglo XIV. En cambio, las localidades costeras de instalación tardía a causa de la presencia de piratas turcos y magrebíes durante la Edad Moderna generan series continuas solo desde las últimas décadas del siglo XVII. 


\begin{tabular}{|l|l|c|c|}
\hline Río/Riera & Localidad & Período & Años \\
\hline Ter/Onyar & Girona & $1322-1971$ & 650 \\
Capaspre & Calella & $1672-1982$ & 311 \\
Sobirans & Arenys & $1687-1982$ & 296 \\
Cirera & Mataró & $1740-1982$ & 243 \\
Besòs & Vallès Occidental & $1402-1971$ & 570 \\
Rieras litorales & Barcelona & $1389-1850$ & 462 \\
Llobregat & El Prat & $1315-1971$ & 657 \\
Francolí & Tarragona & $1595-1930$ & 336 \\
Ebre & Tortosa & $1355-1982$ & 628 \\
Segre & Seu d'Urgell & $1453-1843$ & 391 \\
Segre & Balaguer & $1617-1874$ & 258 \\
Segre & Lleida & $1329-1787$ & 459 \\
\hline
\end{tabular}

TABLA 4. Series de inundaciones utilizadas en el índice general para Catalunya.

\subsection{Información meteorológica instrumental}

Las observaciones meteorológicas instrumentales disponibles en Barcelona ofrecen una cobertura temporal y una calidad que permiten desarrollar múltiples investigaciones en el ámbito de la climatología histórica. Los registros sistemáticos a resolución diaria se iniciaron el 1 de enero de 1780. Hasta mediados del siglo XIX fue una actividad en la que los médicos tenían un especial interés por las circunstancias atmosféricas y su posible relación con el estado sanitario de la población y el desarrollo de episodios epidémicos.

Si bien las series de presión, temperatura y dirección del viento pueden considerarse continuas a resolución diaria, la precipitación presenta unos registros con bastantes interrupciones y una resolución únicamente mensual (ver tabla 5).

La serie fue iniciada por el Dr. Francisco Salvà Campillo, con las observaciones realizadas en su domicilio particular desde 1786 hasta 1827. Las mediciones se realizaban en pulgadas francesas del pie de París. El Dr. Pedro Vieta Gibert, discípulo de Salvà, continuó las observaciones con los mismos métodos entre 1827 y 1843 pero trasladando el observatorio a la sede del «Diario de Barcelona». Las mediciones se realizaban en pulgadas castellanas del pie de Burgos. La década de los 40 supuso una interrupción de la actividad, producida probablemente por los desórdenes sociales e incluso bélicos que sufrió el país en aquellos años.

La serie se reemprende en 1850 con las actividades del matemático Lorenzo Presas, cuyo trabajo como observador aficionado tiene una calidad excepcional. Sus observaciones pueden sustituirse ya en 1860 por los registros realizados por instituciones oficiales con la finalidad específica de realizar y recopilar observaciones meteorológicas instrumentales. Hasta la Guerra Civil las observaciones se realizaron en los dos edificios de la Universidad, antiguo y moderno, con los que finaliza un período en el que los cambios de estación fueron relativamente escasos y con desplazamientos de unos pocos centenares de metros (figura 2). 


\begin{tabular}{|l|l|l|l|}
\hline Observador & Período & Localización & Observaciones \\
\hline F. Salvà & $1786-1827$ & c/ Petritxol, 11 & Documentos manuscritos inéditos $^{1}$ \\
P. Vieta & $1827-1843$ & c/ Llibreteria, 22 & Diario de Barcelona $^{2}$ \\
L. Presas & $1850-1859$ & c/ Sant Pau, 22 & Documentos manuscritos inéditos $^{3}$ \\
Universidad & $1860-1872$ & c/ Carme & Edificio antiguo Universidad $^{\text {Universidad }}$ \\
SMN & $1873-1938$ & Pl. Universidad & Edificio moderno Universidad \\
INM & $1939-1970$ & c/ Travesera de Dalt, 110 & \\
INM & $1971-1975$ & Av. García Morato, 7 & \\
\hline
\end{tabular}

(1): Archivo de la Real Academia de Medicina de Barcelona, F. Salvà, «Tablas Meteorológicas», 3 vols.

(2): Diario de Barcelona. Publicación diaria. Colección completa consultada en el Archivo Histórico de la Ciudad de Barcelona.

(3): Archivo de la Real Academia de Ciencias y Artes, Lorenzo Presas, «Observaciones meteorológicas», 2 legajos.

TABLA 5. Relación cronológica de los observatorios utilizados en la reconstrucción de la serie de precipitación total mensual.

Los datos procedentes de centros oficiales han sido recopilados, tratados y publicados tanto en trabajos de investigación (BURGUENO, 1981), como por el propio Instituto Nacional de Meteorología (ROLDÁN, 1985; INM, 1996).

Los datos del período anterior (1786-1843), esenciales en el solapamiento con los proxy-data procedentes de la documentación histórica, se han obtenido en fuentes documentales y bibliográficas (ver tabla 5 y figura 2).

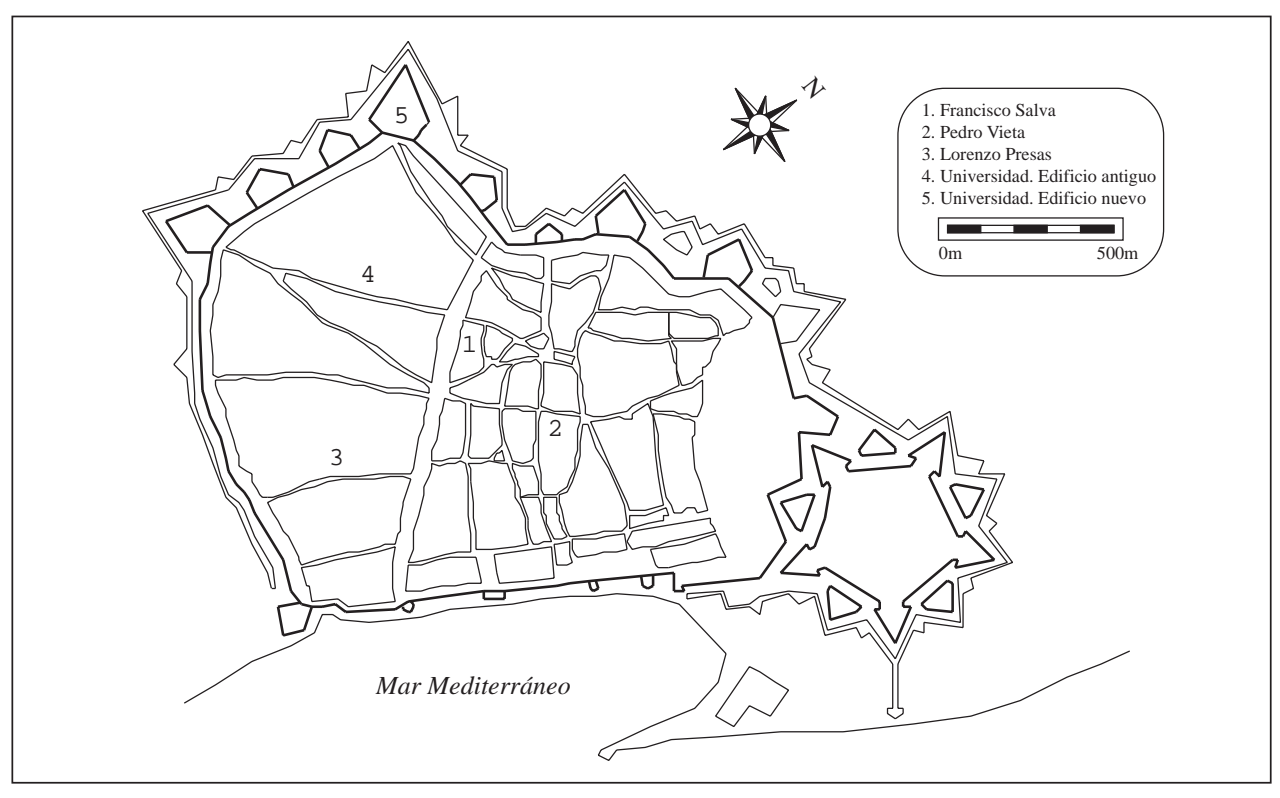

FIGURA 2. Localización de los observadores meteorológicos instrumentales en Barcelona a partir de los cuales se ha establecido la serie de precipitación total mensual. Período antiguo. 


\section{Elaboracion de un índice hídrico para Catalunya a partir de información histórica}

La disponibilidad de datos en series prolongadas de diferentes localizaciones geográficas ha representado un factor positivo en el tratamiento de los datos históricos. La posibilidad de construir unos índices que pudieran sintetizar estas series ofrecía dos aspectos positivos. En primer lugar, se conseguía limitar en lo posible el efecto que cualquier error o falta de homogeneidad en los registros documentales podría introducir en las series de datos. En segundo lugar, se intentaba obviar aquellos comportamientos propios de una variabilidad local, condicionada por la compleja multiplicidad de factores ambientales que intervienen en la caracterización del régimen hídrico y térmico.

Para ello se construyó un solo índice hídrico a partir de todas las series con información proxy-data disponibles. La procedencia variada de sus datos era una garantía de máxima representatividad. Este índice hídrico sería el empleado en el proceso de calibración con la serie de precipitaciones a resolución anual disponible para Barcelona desde 1786 realizada con registros instrumentales.

De entre todos los puntos disponibles, se seleccionaron aquellos que ofrecían una información más rica y detallada, tanto en los aspectos de déficit hídrico como en los episodios de inundaciones. Con este criterio se seleccionaron aquellas localidades más representativas, ofreciendo el siguiente resultado:

$$
\begin{array}{ll}
\text { - Girona } & \text { : tercio norte del territorio catalán } \\
\text { - Barcelona y Tarragona } & : \text { costa central catalana } \\
\text { - Tortosa } & : \text { costa meridional } \\
\text { - Cervera y Lleida } & : \text { sector interior de Catalunya. }
\end{array}
$$

El índice a construir debía reunir dos condiciones. Por un lado, ser representativo; es decir, aportar una información rápida y precisa de las condiciones hídricas que definen un año concreto. En segundo lugar debía ser equitativo; es decir, cada localidad debía aportar al índice la cantidad óptima de información teniendo en cuenta el origen de la serie de precipitación. El índice desarrollado resulta de la integración de los registros para cada localidad y en base a la combinación de dos componentes:

- Un primer componente que define las condiciones de sequía a partir de la frecuencia de las rogativas «pro pluvia» en combinación con su nivel de gravedad (IMCS) y de la duración de los episodios (NdII).

- El segundo componente que define o caracteriza las condiciones de exceso hídrico, empleando para ello el índice de frecuencia de rogativas «pro serenitate» (CR) y el índice de frecuencia de inundaciones (INU).

La generación de un índice basado en múltiples índices previos permite que la información histórica cualitativa de partida pase a tener ya un carácter estrictamente cuantitativo. Sólo con este trabajo previo era posible cumplir el objetivo de generar un mecanismo para la calibración de datos históricos con datos instrumentales, en este caso de precipitación. La construcción del índice ha seguido los pasos siguientes:

$\mathbf{1}^{\mathbf{0}}$ : cuantificación de la información histórica mediante los índices parciales arriba indicados (BARRIENDOS, 1994):

IMCS: suma ponderada de los diferentes niveles de rogativas «pro pluvia» registrados en un año. Se atribuye un valor 1 a las rogativas de nivel I; 2 a las rogativas de nivel II, y así sucesivamente hasta el nivel máximo (V). 
NdII: suma de días por año en los que había estado activado el nivel II de rogativas «pro pluvia» (única ceremonia de este tipo que presenta una duración sostenida durante todo un episodio de sequía).

CR: Ciclos de remisión o rogativas «pro serenitate», ceremonias realizadas cuando las lluvias continuas amenzaban el desarrollo de los cultivos.

INU: frecuencia absoluta anual de ocurrencia de las inundaciones de diferentes intensidades. $2^{\mathbf{0}}$ : estandarización de IMCS, NdII, CR y INU. De este modo todos los índices tienen base 0 y adquieren una comparatividad completa entre variables y entre localidades diferentes. $3^{\mathbf{o}}$ : ponderación de cada uno de los cuatro índices, según la información aportada por cada uno de ellos. A IMCS se le da un valor de «2» y a INU un valor de «1,5». Los dos restantes no se ponderan en ningún sentido.

$4^{\mathbf{0}}$ : combinación de los cuatro índices. Se realiza a partir de una simple suma aritmética pero cambiando de signo los dos que indican sequía: IMCS y NdII.

El resultado es un índice único e individual para cada una de las localidades. Para el caso de Barcelona se presenta del siguiente modo:

$$
\mathrm{IND}_{\mathrm{BAR}}=(1,5 \mathrm{INU}+\mathrm{CR})-(2 \mathrm{IMCS}+\mathrm{NdII})
$$

Para Girona, Tarragona y Tortosa, al no disponer de un registro con la duración de las rogativas «pro pluvia» de nivel II (NdII) quedará:

$$
\mathrm{IND}_{\mathrm{GIR}} ; \mathrm{IND}_{\mathrm{TAR}} ; \mathrm{IND}_{\mathrm{TOR}}=(1,5 \mathrm{INU}+\mathrm{CR})-2 \mathrm{IMCS}
$$

Por último, con Cervera y Lleida se ha formado un solo índice, al no disponer de información completa por sí mismos. Cervera ha proporcionado la serie de rogativas (IMCS, CR) y Lleida ha proporcionado las inundaciones (INU). La forma que adopta el índice, IND $_{\text {INT, }}$ será igual que (2).

$\mathbf{5}^{\mathbf{0}}$ : construcción del índice a nivel de Catalunya. Se consigue mediante la ponderación de cada localidad y la suma aritmética de todas ellas, presentando la siguiente forma:

$$
\mathrm{IND}=\mathrm{A} \mathrm{IND}_{\mathrm{BAR}}+\mathrm{B} \mathrm{IND}_{\mathrm{GIR}}+\mathrm{C} \mathrm{IND}_{\mathrm{INT}}+\mathrm{D} \mathrm{IND}_{\mathrm{TAR}}+\mathrm{EIND}_{\mathrm{TOR}}
$$

Para la estimación de las constantes A, B, C, D y E, se ha optado por encontrar el peso relativo de cada uno de los índices en base a la correlación existente con el de Barcelona. Así, cada peso variará entre 1, si la información aportada es máxima, hasta 0 , si ésta es nula. Evidentemente pueden tener valores negativos si la correlación es negativa. Desarrollando el método y sustituyendo en (3) los coeficientes respectivos, el índice hídrico anual para Catalunya se constituiría mediante la fórmula:

$$
\mathrm{IND}=\mathrm{IND}_{\mathrm{BAR}}+0,28 \mathrm{IND}_{\mathrm{GIR}}+0,26 \mathrm{IND}_{\mathrm{INT}}+0,24 \mathrm{IND}_{\mathrm{TAR}}+0,21 \mathrm{IND}_{\mathrm{TOR}}
$$

Este índice se ha calculado para el período común que se extiende entre 1521 y 1850, indicando años secos cuando el IND es menor que cero y años húmedos cuando es mayor que cero, y la intensidad del fenómeno, cuanto más alto sea su valor (tanto positivo como negativo). 


\section{Obtención de resultados}

\subsection{Modelo de regresión}

El resultado obtenido con los índices se puso en comparación con la información de procedencia instrumental. El objetivo era elaborar un modelo que permitiera la calibración de la información histórica (IND) a partir de la precipitación total anual de la serie de Barcelona. Con ello, se pretendía conseguir una reconstrucción teórica de la serie anual pluviométrica entre 1521 y 1786 , permitiendo así finalmente disponer de una serie continua con valores instrumentales para la ciudad de Barcelona desde 1521 hasta la actualidad.

El período óptimo de calibración se situó entre 1787 (año que empiezan los registros pluviométricos instrumentales) y 1850 (límite máximo del buen funcionamiento del sistema de rogativas en la ciudad de Barcelona). De este período de solapamiento de ambos tipos de información, se han utilizado solo aquellos años que disponen de información continua para los doce meses, despreciando el resto.

Igualmente, se ha analizado cada uno de los años a través de un correlograma (figura 3). La correlación lineal, año a año, entre IND y la precipitación registrada muestra como la correlación es significativa hasta 1837, cayendo después de forma ostensible.

\begin{tabular}{|c|c|c|c|c|c|c|c|}
\hline AÑO & PREP & IND & $\mathbf{r}$ & AÑ & PREP & IND & $\mathbf{r}$ \\
\hline 1787 & 568,5 & 3,36 & & 1814 & 508,7 & 0,15 & 0,80 \\
1788 & 663,2 & 5,15 & 1,00 & 1815 & 383,5 & $-0,95$ & 0,81 \\
1789 & 310,2 & $-0,53$ & 1,00 & 1816 & 295,5 & $-2,52$ & 0,84 \\
1790 & 765,8 & 5,30 & 0,98 & 1817 & 196,3 & $-12,00$ & 0,80 \\
1791 & 538,1 & 1,86 & 0,97 & 1818 & 460,2 & 0,26 & 0,80 \\
1793 & 670,0 & 4,27 & 0,97 & 1819 & 573,0 & 0,66 & 0,79 \\
1794 & 594,4 & 3,60 & 0,97 & 1820 & 654,2 & 3,82 & 0,80 \\
1795 & 504,2 & 0,51 & 0,94 & 1821 & 577,5 & 0,13 & 0,79 \\
1796 & 486,1 & 0,66 & 0,94 & 1822 & 264,0 & $-4,91$ & 0,81 \\
1797 & 424,1 & 1,01 & 0,94 & 1823 & 215,4 & 0,66 & 0,76 \\
1800 & 548,2 & 0,35 & 0,89 & 1824 & 266,2 & $-3,13$ & 0,77 \\
1801 & 670,0 & 2,09 & 0,85 & 1826 & 647,4 & 0,66 & 0,76 \\
1802 & 472,6 & 0,55 & 0,86 & 1827 & 719,8 & 3,82 & 0,77 \\
1803 & 507,6 & 0,07 & 0,85 & 1837 & 677,2 & 5,05 & 0,78 \\
1805 & 543,7 & 0,35 & 0,83 & 1838 & 957,8 & 0,55 & 0,69 \\
1806 & 492,9 & 0,66 & 0,83 & 1839 & 758,5 & 0,48 & 0,67 \\
1807 & 459,7 & $-0,68$ & 0,84 & 1840 & 892,0 & 0,47 & 0,63 \\
1812 & 286,5 & $-0,92$ & 0,84 & 1850 & 983,2 & 1,21 & 0,59 \\
1813 & 259,4 & 0,24 & 0,81 & & & & \\
\hline
\end{tabular}

TABla 6. Período óptimo utilizado para la calibración, valores registrados instrumentalmente, a través de proxy-data (índice IND) y su correlación. 


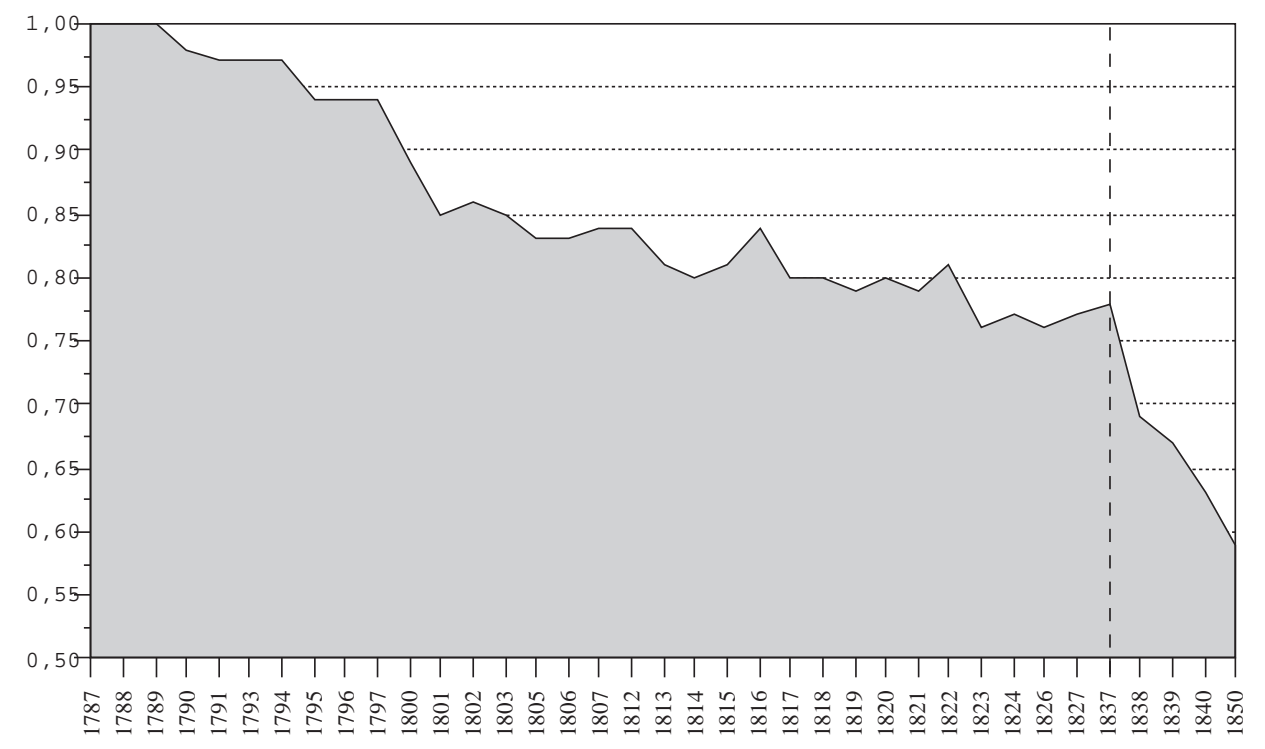

FIGURA 3. Correlograma 1787-1850. Coeficiente de correlación de Pearson de la serie de precipitación total anual de Barcelona.

La pérdida de fiabilidad en las décadas centrales del siglo XIX es fácilmente atribuible a aspectos históricos. Especialmente, a los conflictos sociales y bélicos que padeció España y que en las grandes poblaciones alteró seriamente algunas actividades culturales y religiosas tradicionales, como eran las ceremonias de rogativas. De hecho, en la ciudad de Barcelona empiezan a aparecer serias dificultades para la realización de rogativas públicas en una fecha bastante temprana. Existe un testimonio directo de este proceso, con una actitud contradictoria, pues mientras defiende como eclesiástico la bondad de las rogativas, disponía a la vez de un barómetro con el que seguir instrumentalmente los avatares meteorológicos:

«Casi toda esta pasada noche empesando a las 10 dadas ha llovido con bastante suavidad, aunqe no con abundancia. Parece que el Cielo ha oido benigno las suplicas qe el Pueblo Barcelones a dirigido al Altisimo por intercesion de Sta. Madrona. A la verdad ha sido este favor tanto mas apreciable por la prontitud, quanto si se hubiera diferido algunos dias hubieran sin duda aguzado sus satíricas y mordaces lenguas aquellos incredulos, materialistas, y toda la chusma de irreligionarios de qe abunda esta Capital, y qe se burlan de la Protección de los Santos. Dicterios bien picantes y malignos se han oido en el decurso de la Procesion haciendo chacota diciendo: Mirad mirad ya llueve en las montañas vecinas. Con este prontisimo socorro de la lluvia, al paso qe estos han quedado confundidos, los verdaderos fieles (de qe abunda Barcelona, y ellos son los qe la sostienen) se han arraigaran (sic) mas, y mas en la Devocion a los Patronos.» (Biblioteca de la Universidad de Barcelona, Ms. 1802, R. FERRER, «Barcelona Cautiva», vol. I, 9/4/1812).

«an salido de la Parroq de S. Jayme las Rogativas para la Catedral a fin de obtener la lluvia competente, la qe no hemos logrado desde el 11 por la noche. El Barometro se 
mantiene todavia en Variable.» (BUB, Ms. 1802, R. FERRER, «Barcelona Cautiva», vol. I, 14/4/1812).

«Ha llovido esta mañana abundantisimamente a Dios gras. de manera qe el Barometro señalaba Aguaceros. Por la tarde antes de las Rogativas ha continuado con suavidad...» (BUB, Ms. 1803, R. FERRER, «Barcelona Cautiva», vol. II, 18/4/1812)

Conocida la serie de datos, el modelo debía preveer que la precipitación, como elemento climático, presenta una acotación tanto inferior como superior al ser poco probable que la precipitación rebase esos umbrales definidos por las condiciones medias atmosféricas. Así, el mejor ajuste de los datos, se ha conseguido mediante la denominada curva logística, la cual permite acotar la función a partir de un límite inferior y del rango máximo en que pueden variar los datos de la variable dependiente.

El ajuste se ha realizado por mínimos cuadrados, resultando la siguiente ecuación:

$$
\mathrm{PREP}=100+\frac{120 \circledast\left[\mathrm{e}^{(\mathrm{IND}-1.571480) \beta}\right]^{021141}}{\left[\mathrm{e}^{(\mathrm{IND}-1.571480) \beta}\right]^{021141}+1.779450}
$$

El coeficiente de correlación que ofrece el modelo es de 0.83 , mientras que la cantidad de información que queda explicada es del 69\%. El ajuste (figura 4) muestra la correlación entre el índice hídrico y la precipitación, así como entre los datos observados y los datos calculados (figura 5).

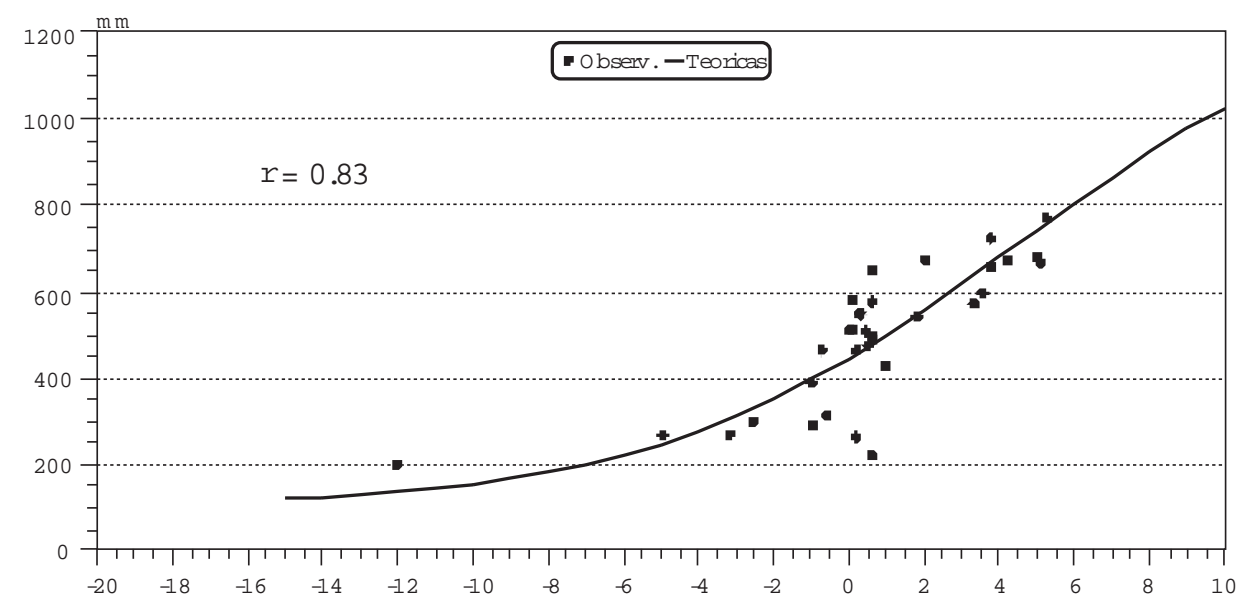

FIGURA 4. Modelo de regresión. IND/PREP. 


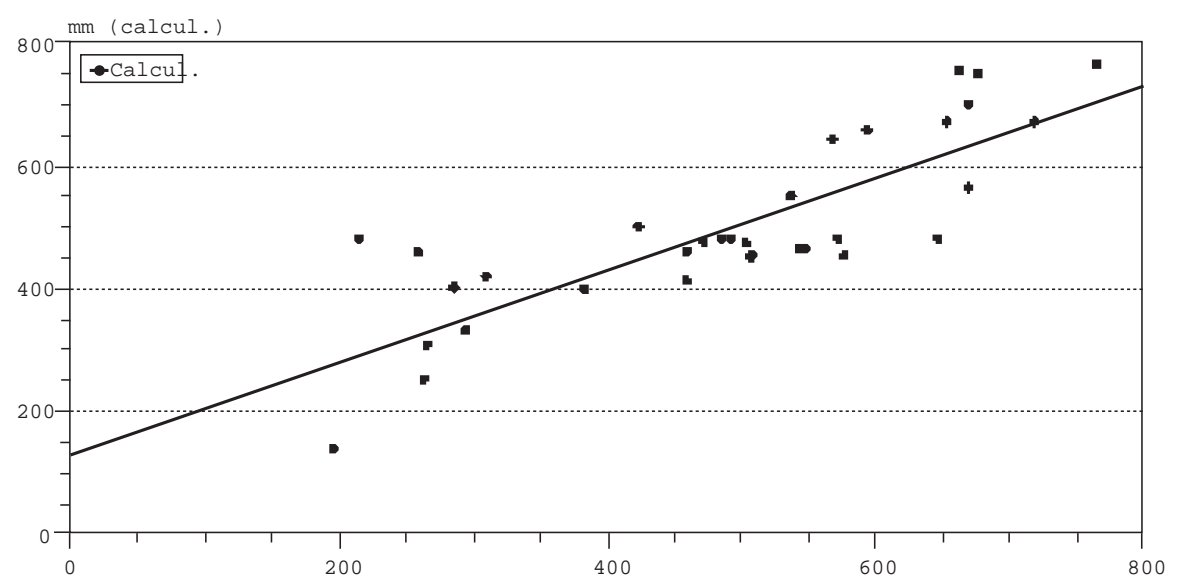

FIGURA 5. Modelo de regresión. Datos observados/Datos calculados.

4.2. Resultados

El modelo de regresión se ha utilizado para estimar la precipitación dentro del período histórico carente de observaciones instrumentales. El resultado de la estimación, con el período posterior disponible en registros instrumentales directos, se ha conjuntado en una sola serie que comprende el período 1521-1989 (figura 6). A primera vista, la gráfica obtenida presenta una tendencia creciente, especialmente dentro del período estimado, que suscita dudas o sospechas de su carácter natural. Para llegar a un resultado más consistente, se decidió homogeneizar toda la serie, pero sin destruir completamente esta tendencia ya que en última instancia podía ser debida realmente a causas climáticas.

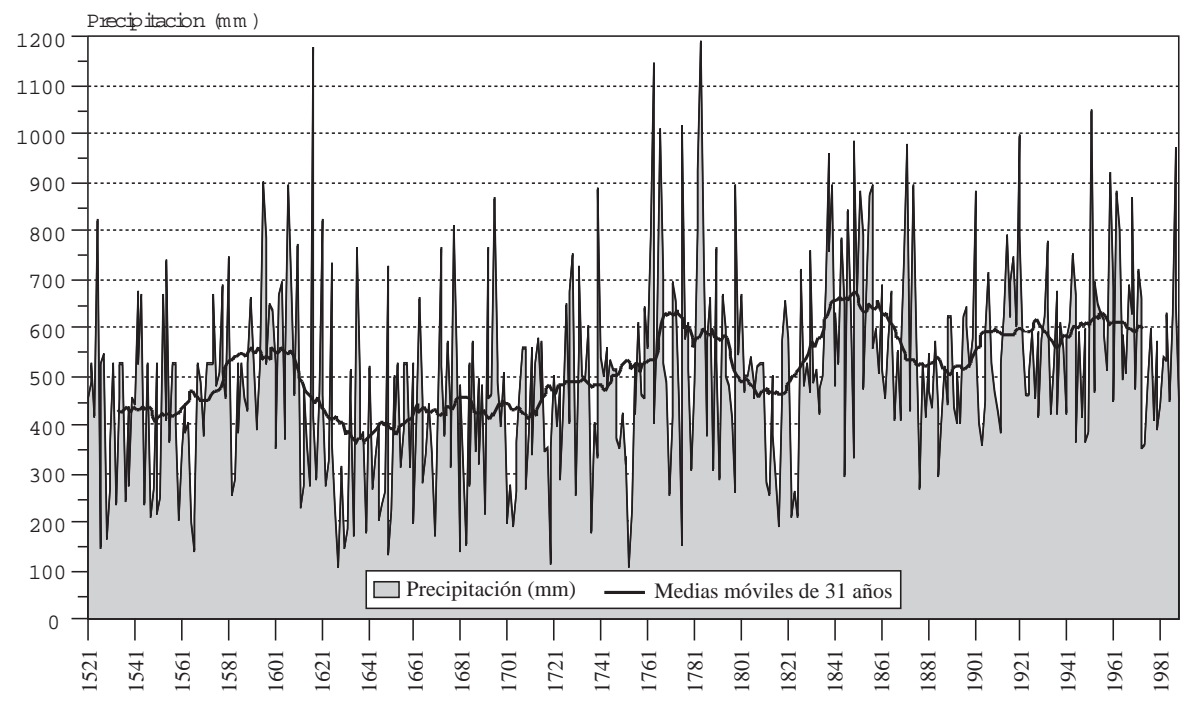

FIGURA 6. Serie de precipitación de Barcelona. Primeros resultados. 
El test de Von Neumann da un valor de 1.5 para la serie completa (figura 7), el cual, aún sin alejarse del umbral 2, obliga a un proceso de homogeneización.

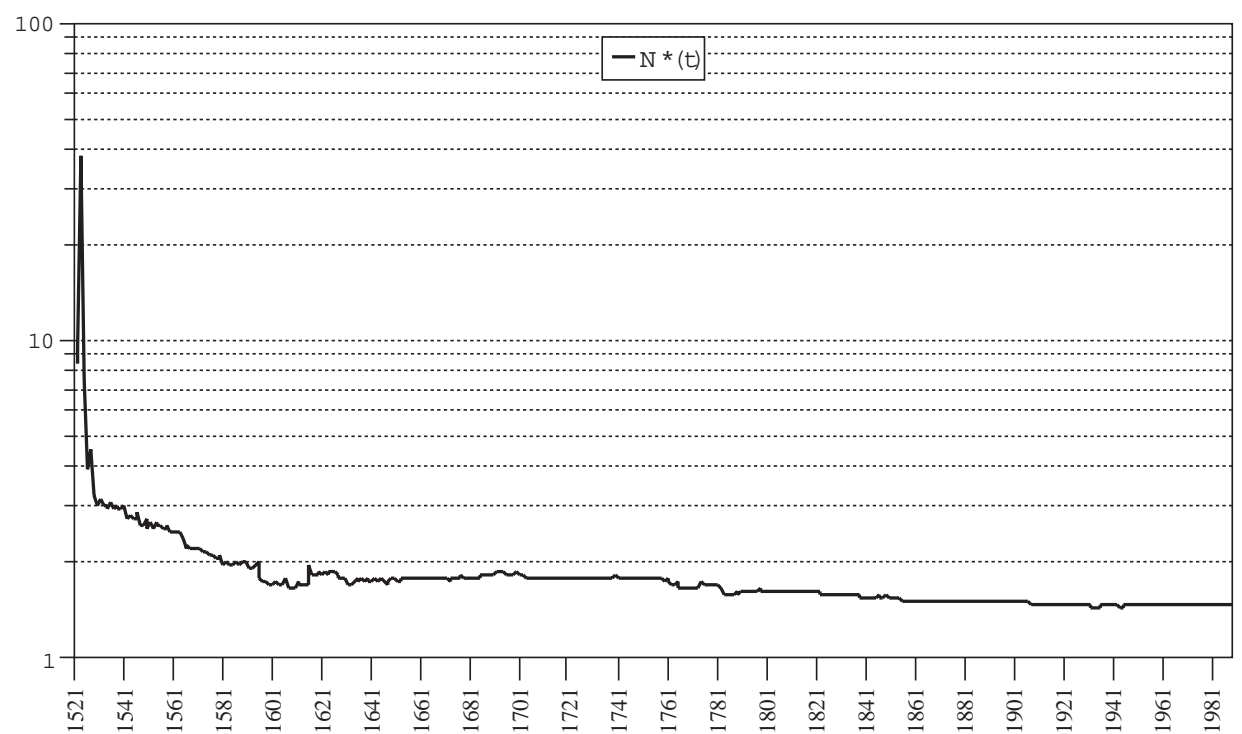

FIGURA 7. Test de Von Neumann para la serie original.

La homogeneización de la serie se realiza utilizando el test de las frecuencias acumuladas (Rodríguez et al., 1996) por dos ventajas que presenta sobre otros de características similares: 1) Se trata de un test de homoegeneidad con un carácter absoluto. Las inhomogeneidades se detectan tomando como base el propio observatorio y, por tanto, se evita la «contaminación» de la serie analizada con información procedente de series externas.

2) Ofrece la posibilidad de datar y cuantificar los «break-points» o inhomogeneidades que aparecen en la serie, en base al cambio de pendiente o signo que se detecta.

El resultado (figura 8) permite la identificación de un «break» principal hacia mediados del siglo XVIII, exactamente en el año 1756. Este cambio de tendencia era presumible, ya que de forma general coincide con el inicio de la serie de valores instrumentales. Esto produce una inhomogeneidad ya intrínseca al método de calibración utilizado. Desde este punto de vista, puede ser mas significativo el «break» que aparece a finales del siglo XVI y primer tercio del siglo XVII ya que es una inhomogeneidad relacionada con los datos históricos. La serie final corregida (figura 9) y su test de frecuencias acumuladas (figura 10) muestran un comportamiento más homogéneo, quedando en evidencia, sin embargo, una inhomogeneidad importante entre las últimas décadas del siglo XVIII y las primeras del siglo XIX. Este comportamiento anómalo tiene casi con toda seguridad un origen natural: todas las informaciones de origen documental recopiladas permiten identificar y caracterizar una oscilación climática, denominada Oscilación Maldà para el caso de Catalunya (BARRIENDOS, 1994). La evidencia de esta oscilación, así como la presencia de un buen número de pequeñas pulsaciones, con un probable origen natural, no se han homogeneizado a fin de preservar sus características para interpretaciones climáticas en investigaciones posteriores. 
Sobre la serie corregida se ha vuelto a aplicar el test de Von Neumann (figura 11), el cual ha alcanzado un valor de 1.7. Por lo tanto, ha mejorado en dos décimas el test anterior realizado para la serie original.

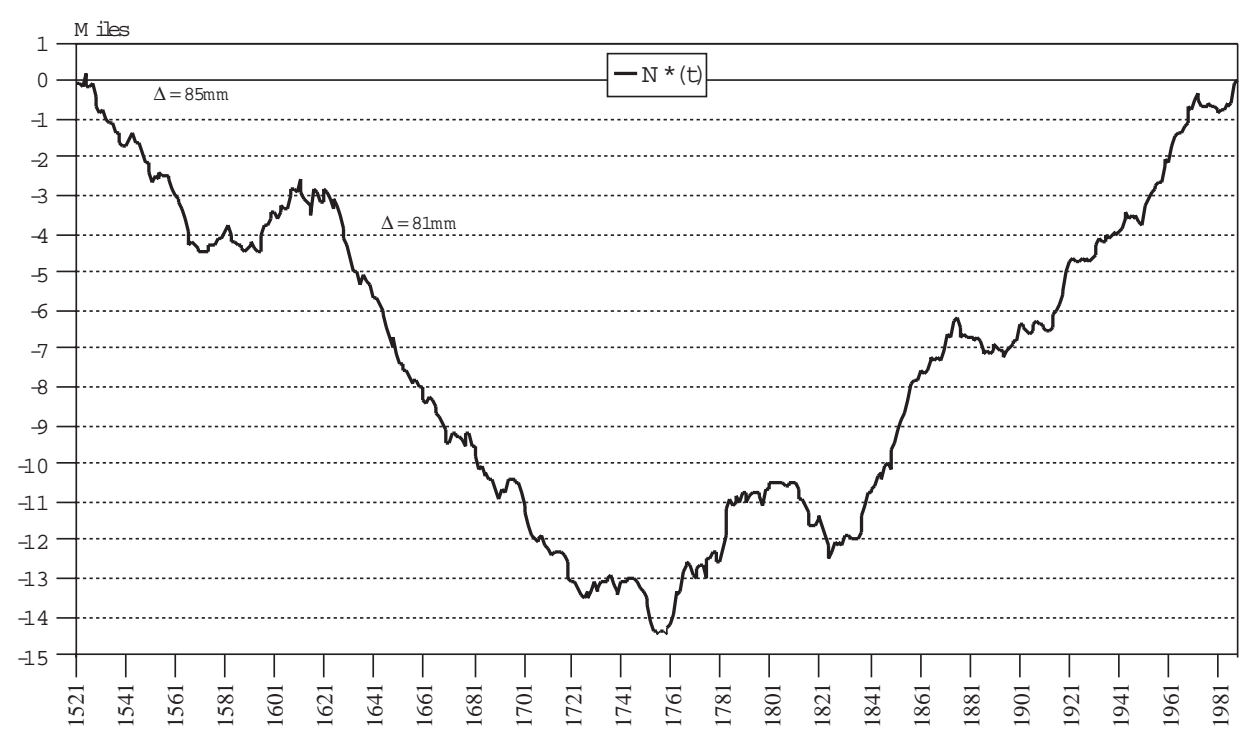

FIGURA 8. Frecuencias acumuladas. Test de R. Rodríguez (1521-1989).

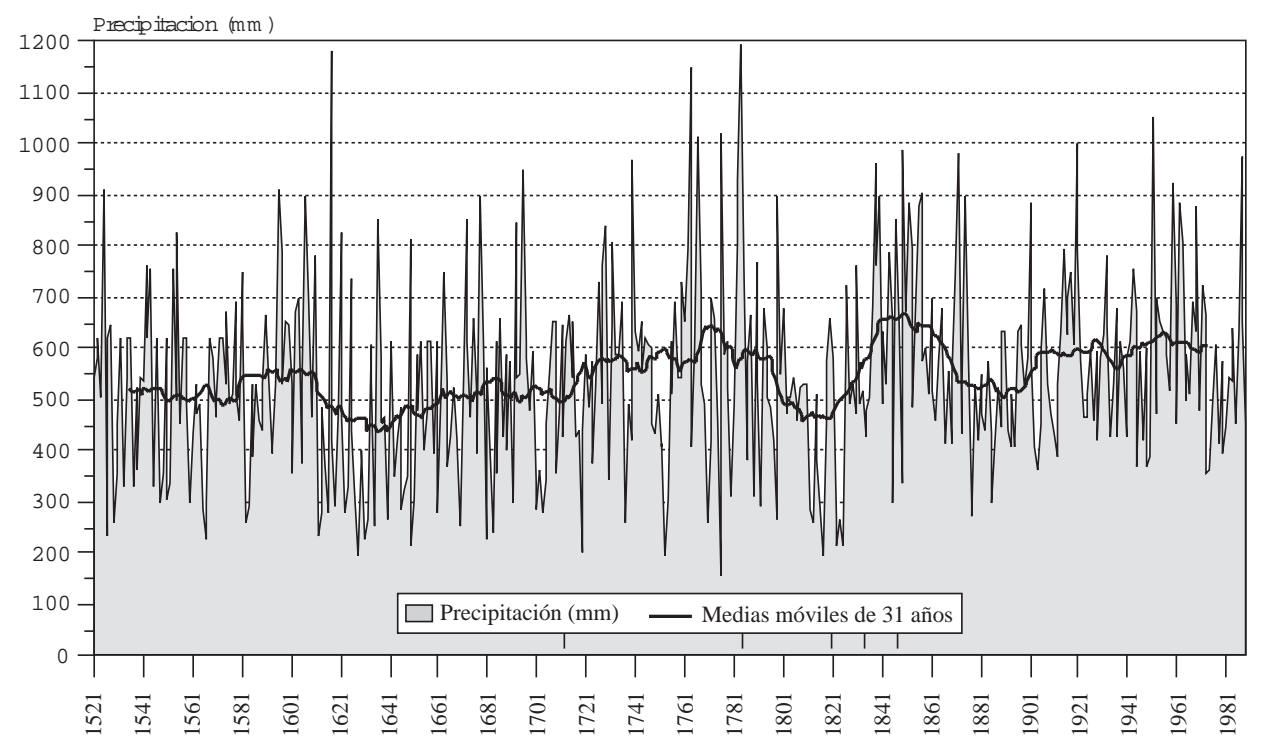

FIGURA 9. Serie de Barcelona corregida (1521-1989). 


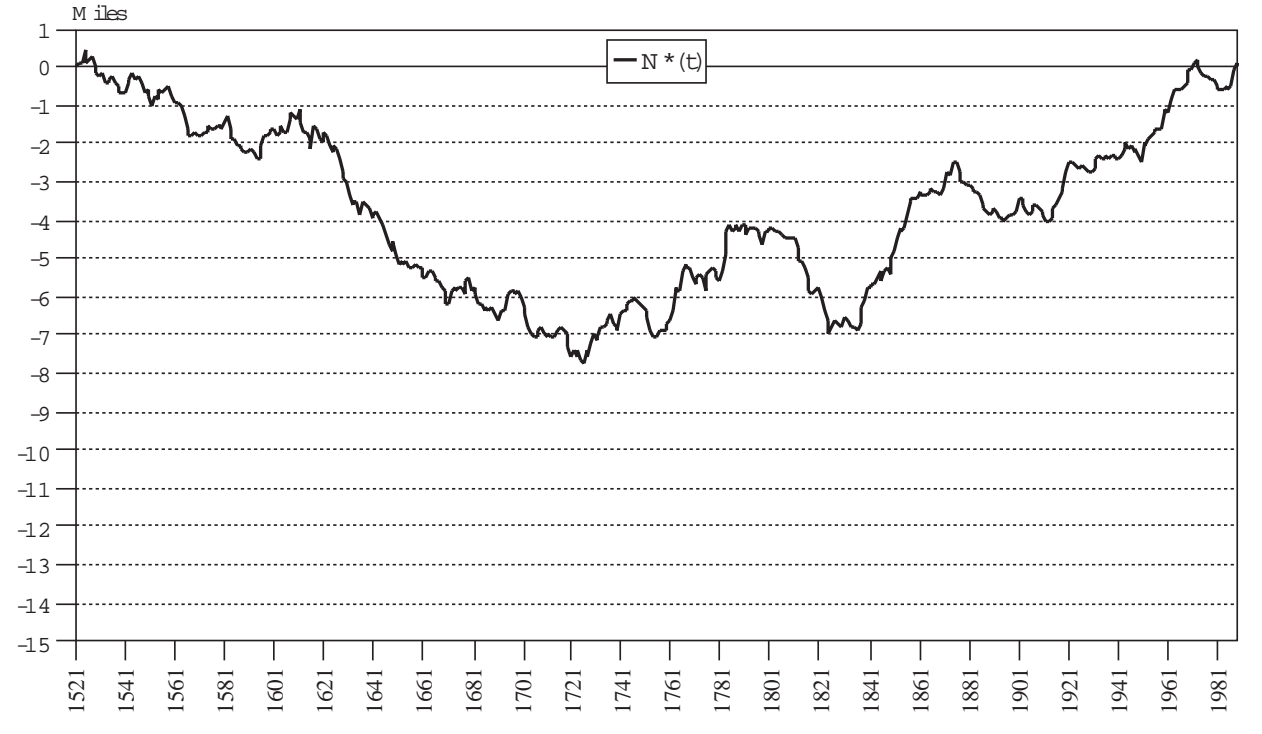

Figura 10. Frecuencias acumuladas. Serie corregida. Test de R. Rodríguez (1521-1989).

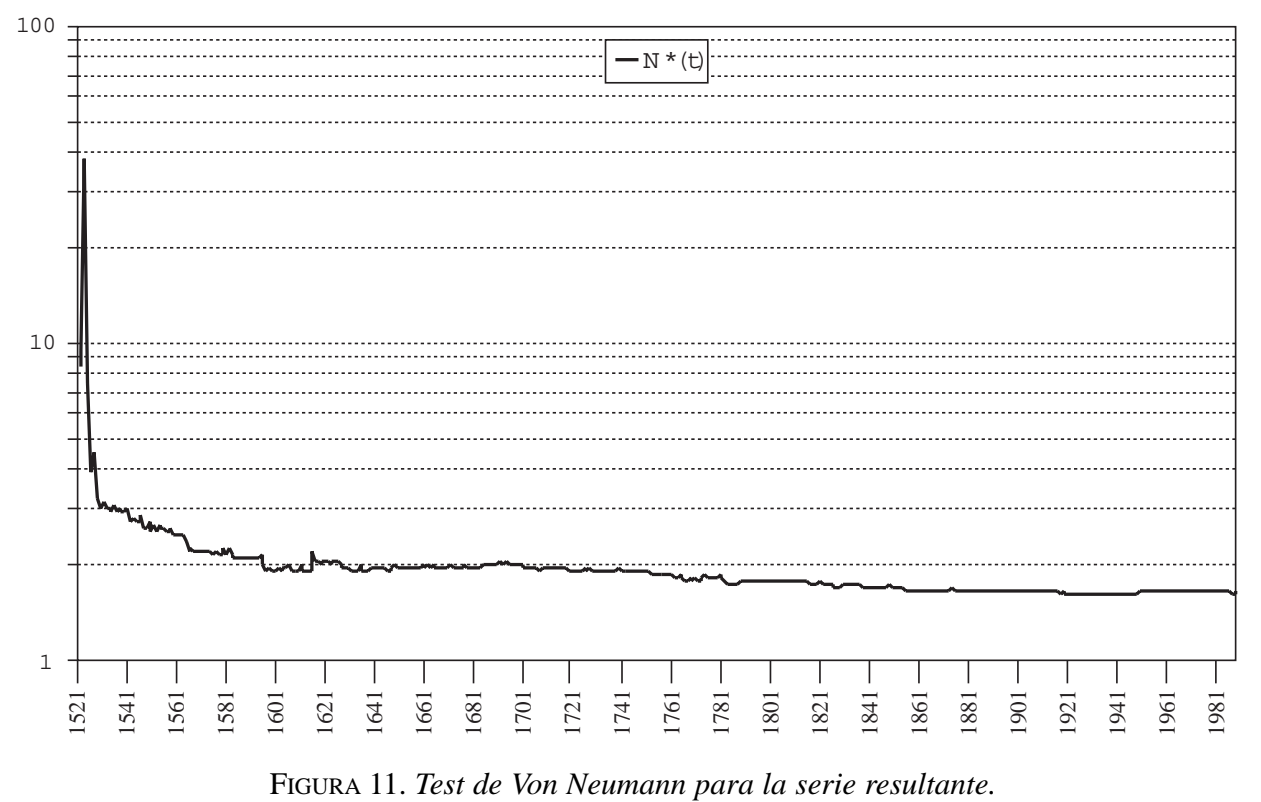




\section{Conclusiones}

La metodología desarrollada en este trabajo a modo de ensayo para su implementación en el tratamiento de series climáticas procedentes de fuentes documentales históricas ofrece una herramienta consistente para cubrir una de las fases más delicadas del trabajo en climatología histórica: la calibración de datos. Por otra parte, se trata de una pauta de trabajo ya empleada en otras especialidades de la paleoclimatología, lo que obliga en cierto modo a su desarrollo y aplicación si se pretende a medio plazo abrir la posibilidad de generar análisis comparativos, por ejemplo, entre la dendroclimatología y la climatología histórica.

Realmente este trabajo metodológico no suscita la elaboración de unas conclusiones determinantes, sino más bien la apertura de nuevas líneas de investigación y la posibilidad de profundizar el tratamiento de algunas series de datos ya disponibles en diferentes grupos de investigación paleoclimática.

Sólo cabría indicar dos aspectos técnicos sobre la disponibilidad de los datos y su tratamiento. En primer lugar, es conveniente indicar que las series obtenidas a partir de registros de ceremonias de rogativas constituyen un buen fondo de datos, tanto por su homogeneidad como por su duración temporal. El hecho de que la serie de Barcelona sea excepcionalmente breve (poco más de 300 años) es resultado directo y casi único de las transformaciones sociales y políticas sufridas por la ciudad a mediados del siglo XIX. Por la experiencia ya recogida en otras poblaciones, se puede asegurar con certeza que en poblaciones más vinculadas a la actividad agraria y con un contexto social más estable se pueden generar series de ceremonias de rogativas con una calidad óptima hasta la Guerra Civil (1936-1939). En todo caso, las dificultades para obtener entonces unos solapamientos prolongados proceden de la tardanza en iniciarse los registros meteorológicos instrumentales en estas poblaciones. En definitiva, parece que el período 1860-1920 puede ofrecer un solapamiento consistente en buena parte de las ciudades capitales de provincia españolas.

En segundo lugar, y para finalizar este apartado, el estudio a resolución anual que se ha presentado como ensayo metodológico presenta por si mismo la evidencia de que es necesario emprender este tipo de investigaciones a unas resoluciones temporales más detalladas. El régimen pluviométrico regular existente en otros ámbitos climáticos, como el Norte de Europa, quizás permitirían la utilización de las resoluciones anuales. Sin embargo, en el clima mediterráneo la irregularidad de su régimen pluviométrico impide confiar en esta resolución. Como bien es sabido, un valor anual de $500 \mathrm{~mm}$ en casi cualquier punto de la Península Ibérica puede proceder tanto de 80 días de precipitación modesta y relativamente regular como de 4 grandes episodios tempestuosos.

Por todo ello parece necesario emprender las investigaciones en resoluciones más altas, aunque sin llegar a un excesivo nivel de detalle, porque entonces la interpretación de los proxy-data de origen documental puede plantear problemas. En el caso de las rogativas «pro pluvia», por ejemplo, la resolución para emprender cada rogativa está perfectamente datada en los documentos, pero realmente responde a una situación de sequía creciente que puede proceder de unos meses anteriores al día mismo de la resolución.

En consecuencia, parece preferible mantener una resolución de trabajo intermedia, como sería la resolución estacional, en vez de mantener la resolución anual o pretender trabajar a niveles de mayor detalle, como sería la resolución mensual. 


\section{Bibliografía}

ALEXANDRE, P.: 1987, Le climat en Europe au moyen âge, Ecole des Hautes Etudes en Sciences Sociales, París.

BARRIENDOS, M.: 1994, El clima histórico de Catalunya. Aproximación a sus características generales (Siglos XV-XIX), Departamento de Geografía Física y AGR, Universidad de Barcelona, 500 pp.

BARRIENDOS, M. y POMES, J.: 1993, L'aigua a Mataró. Inundacions i recursos hídrics (ss. XVIII-XX), Caixa d'Estalvis Laietana, Mataró.

BURGUEÑO, A.: 1981, Diversos aspectos climatológicos de la lluvia en Barcelona Tesina de licenciatura, Departamento de Física de la Tierra y el Cosmos, Universidad de Barcelona.

CODINA VILA, J.: 1971, Inundacions al Delta del Llobregat, R. Dalmau, Barcelona, Col «Episodis de la Histbria», nos. 147-148.

CREUS, J.; FERNÁNDEZ CANCIO, A. Y MANRIQUE, E.: 1996, «Evolución de la temperatura y precipitación anuales desde el año 1400 en el sector central de la depresión del Ebro», Lucas Mallada. Revista de Ciencias, Instituto de Estudios Altoaragoneses, 8, 9-27.

CREUS, J.; FERNÁNDEZ CANCIO, A. Y MANRIQUE, E.: 1997, «Dendrocronología y clima del último milenio en España. Aspectos metodológicos y avance de resultados», en Ibáñez, J.J.; Valero Garcés, B.L. y Machado, C. (eds.): El paisaje mediterráneo a través del espacio y del tiempo. Implicaciones en la desertificación, Geoforma Ediciones, Logroño, pp. 311-330.

FRENZEL, B. (ed.): 1994, Climatic trends and anomalies in Europe. 1675-1715, European Science Foundation, Strasbourg, Paläoklimaforschung, 13.

GLASER, R. \& WALSH, R. (eds.): 1991, Historical Climatology in Different Climatic Zones, Würzburger Geographische Arbeiten, 80, Universität Würzburg.

INM: 1996, Homogeneidad y variabilidad de los registros históricos de precipitación de España, Instituto Nacional de Meteorología, Madrid.

LE ROY LADURIE, E.: 1967, Histoire du climat depuis l'an mil, Flammarion, París.

MARTÍN VIDE, J. y BARRIENDOS, M.: 1995, «The use of rogation ceremony records in climatic reconstruction: a case study from Catalonia (Spain)», Climatic Change, 30, Kluwer Academic Publishers, pp. 201-221.

PFISTER, C.: 1988, Klimageschichte der Schweiz, 1525-1860. Das Klima der Schweiz von 1525-1860 und seine Bedeutung in der Geschichte von Bevölkerung und Landwirtschaft, Paul Haupt, Bern.

RODRÍGUEZ, R.; LLASAT, M.C. y MARTÍN VIDE, J.: 1996, «Revisión de los criterios de homogeneidad aplicados a variables meteorológicas», en JUARISTI, J. Modelos y sistemas de información en Geografía, Universidad del País Vasco, Vitoria, pp. 98-112.

ROLDÁN, A.: 1985, Notas para una climatología de Barcelona, Instituto Nacional de Meteorología, Madrid.

SANZ, M.: 1983, El Ripoll i les seves avingudes, Col.legi Oficial de Llicenciats i Doctors de Catalunya i Balears, Sabadell. 


\section{Agradecimientos}

- Proyecto Annual to Decadal Variability In Climate in Europe (ADVICE), ENV4CT95-0129-PL951090, 1996-1997, Comisión Europea.

- Proyecto Variabilidad natural anual y por décadas en el clima de la Península Ibérica, CLI95-1928-C02-02, CICYT, España. 